ARTIGO ORIGINAL

\title{
Avaliação de assimetria hemisférica funcional em pacientes com doença de Alzheimer
}

\author{
Evaluation of functional hemispheric asymmetry in Alzheimer's disease patients
}

\author{
Tandara Oliveira Benevides Silva ${ }^{1}$, Albert C. Yang ${ }^{2}$, Eduardo Pondé de Sena ${ }^{3 *}$ \\ ${ }^{1}$ Mestranda em Processos Interativos de Órgãos e Sistemas pela Universidade Federal da Bahia (UFBA); ${ }^{2}$ \\ Departamento de Psiquiatria do Hospital Geral de Veteranos de Taipei, Taiwan; Beth Israel Deaconess Medical \\ Center, Harvard Medical School; ${ }^{3}$ Professor Associado de Farmacologia e Terapêutica do Departamento de Biorregulação do \\ Instituto de Ciências da Saúde da UFBA.
}

\begin{abstract}
Resumo
Introdução: as assimetrias cerebrais constituem aspectos fundamentais para a organização funcional e o processamento cognitivo. Estudos prévios têm reportado que alterações no padrão de assimetria hemisférica existentes em algumas patologias reduzem a eficiência no processamento de informação. Objetivos: avaliar a assimetria hemisférica funcional a partir de padrões de homofilia e heterofilia; analisar a assimetria da conectividade funcional de redes dinâmicas em portadores de Alzheimer. Metodologia: foram construídas redes funcionais dinâmicas do córtex cerebral de pacientes com diferentes níveis de Alzheimer (muito leve, leve e moderado a grave) e do grupo de controle a partir de EEG (19 eletrodos, Sistema 10-20). Tais redes foram geradas a partir do método de associação sincronização por motifs e representadas através de grafos variantes no tempo e de redes estáticas agregadas. Essas redes foram avaliadas por meio de índices de assimetria e conectividade. Resultados: os resultados mostram uma tendência à redução da homofilia com a progressão da enfermidade, quando se compara ao controle, além de um leve aumento do índice para o último estágio, se comparado aos estágios iniciais da doença. A assimetria para a conectividade funcional, por sua vez, mostrou uma propensão a maior conectividade para o hemisfério direito, salvo para o estágio moderado a grave. Conclusão: embora os resultados não tenham apresentado diferenças significativas, eles indicam tendências de alteração no padrão de homofilia e na assimetria da conectividade funcional em redes funcionais dinâmicas de pacientes com Alzheimer.

Palavras-chave: Doença de Alzheimer. Assimetria. Conectividade funcional. Homofilia.
\end{abstract}

\begin{abstract}
Introduction: brain asymmetries are fundamental aspects for functional organization and cognitive processing. Previous studies have reported that changes in the hemispheric asymmetry pattern of some pathologies reduce the efficiency of information processing. Objectives: to evaluate functional hemispheric asymmetry based on homophilia and heterophilia patterns; to analyze the asymmetry of functional connectivity of dynamic networks in Alzheimer patients. Methodology: dynamic functional networks of the cerebral cortex were constructed from patients with different levels of Alzheimer's (very mild, mild and moderate to severe) and the control group from EEG (19 electrodes, System 10-20). Such networks were generated from the motif synchronization association method and represented by time-varying graphs and aggregate static networks. These networks were evaluated using asymmetry and connectivity indices. Results: the results show a tendency towards a reduction in homophilia with disease progression, when compared to the control, besides a slight increase in the index for the last stage, compared to the initial stages of the disease. Asymmetry for functional connectivity, in turn, showed a propensity for greater connectivity to the right hemisphere, except for the moderate to severe stage. Conclusion: although results did not show significant differences, they indicate trends of change in homophily pattern and functional connectivity asymmetry in dynamic functional networks of patients with Alzheimer's.

Keywords: Alzheimer's Disease; Asymmetry; Functional Connectivity; Homophilia
\end{abstract}

\section{INTRODUÇÃO}

$O$ termo assimetria refere-se às diferenças entre regiões cerebrais correspondentes nos dois hemisférios, e pode ou não ser direcionada para um deles; o processo de assimetria dirigida tem sido denominado lateralização

Correspondente/Corresponding: *Eduardo Pondé de Sena - Universidade Federal da Bahia, Instituto de Ciência e Saúde, Departamento de Biorregulação, End: Avenida Reitor Miguel Calmon s/n, Vale do Canela, CEP: 40.1101100 - Salvador, BA - Tel: (71) 3283-8908 - E-mail: eduardoponde@hotmail.com hemisférica ${ }^{1}$. A presença de assimetria hemisférica é um princípio fundamental da constituição do sistema nervoso central (SNC) e modela a organização funcional da maioria dos sistemas cognitivos ${ }^{2,3}$. Humanos e outros animais exibem padrões de assimetria cerebral que podem ser visualizados nos âmbitos estrutural e funcional ${ }^{4}$.

Estima-se que padrões assimétricos apresentem caráter adaptativo e tenham sido desenvolvidos como respostas importantes ao longo do processo evolutivo $0^{4,5}$. Assim, assimetrias de regiões cerebrais no funcionamento 
da linguagem, em tarefa visuoespacial e em diversos aspectos da emoção são relacionadas ao processamento ótimo da informação e constituem aspectos fundamentais para o desenvolvimento humano ${ }^{4,5}$.

Sabe-se que a assimetria cerebral sofre variações ao longo do processo de envelhecimento humano e durante o desenvolvimento de algumas patologias ${ }^{5-7}$. Estudos prévios com comparações morfométricas obtidas a partir de exames de neuroimagem e, mais recentemente, com o emprego da abordagem de grafos têm demonstrado alterações nas assimetrias estrutural e funcional no cérebro de pacientes com demência ${ }^{1,5,8}$.

Apesar da existência de padrões assimétricos em diversos níveis de análise cerebral, na anatomia, na neuroquímica e na citoarquitetura, por exemplo ${ }^{9}$, e da relação de influência entre assimetrias estruturais, como a do corpo caloso, substância cinzenta e substância branca no desenvolvimento de assimetrias hemisféricas funcionais ${ }^{3}$, este trabalho se dedica apenas ao estudo de assimetrias hemisféricas funcionais, sob a perspectiva de grafos dinâmicos.

O cérebro é um sistema complexo e pode ser representado através da abordagem de grafos ${ }^{10}$. Os grafos são formados por um conjunto de elementos (nós, vértices) e as interações entre eles (arestas), e seu significado depende do que está sendo modelado ${ }^{10,11}$. Neste trabalho, os grafos variantes no tempo (GVT) são usados para caracterizar a conectividade funcional dinâmica. Essa ferramenta de análise permite a avaliação do cérebro como uma rede complexa dinâmica, abordando elementos que as análises convencionais, embasadas no espectro de frequência, não são capazes de avaliar ${ }^{11,12}$.

Estudos recentes, que fazem a análise da assimetria cerebral sob a perspectiva de redes, têm relatado alterações no padrão normal de assimetria, estrutural e funcional, em uma série de transtornos do neurodesenvolvimento e psiquiátricos, como esquizofrenia ${ }^{13}$, epilep$\mathrm{sia}^{14}$, transtorno de déficit de atenção e hiperatividade ${ }^{15} \mathrm{e}$ doença de Alzheimer (DA) ${ }^{8}$. Tais alterações geram danos principalmente na eficiência da rede, o que leva à perda do processamento ótimo da informação, característica da topologia de redes cerebrais ${ }^{8}$.

A expressão assimetria hemisférica funcional (AHF) é empregada para caracterizar distinções funcionais entre os hemisférios direito e esquerdo do cérebro ${ }^{3}$. As AHF têm sido visualizadas em diversos domínio cognitivos, como atenção espacial ${ }^{16}$, linguagem ${ }^{9}$, memória ${ }^{17}$ e funções cognitivas que aparecem geralmente danificadas durante o desenvolvimento da DA.

Shang et al. ${ }^{18}$ demonstraram a existência de AHF em redes funcionais de ressonância magnética funcional de indivíduos saudáveis, com coeficiente de aglomeração, comprimento de caminho e modularidade apresentando assimetria para a direita, enquanto a eficiência global revelou assimetria para a esquerda. Em pacientes com DA, têm sido encontradas diversas modificações em índices de redes funcionais, como perda da eficiência global da rede ${ }^{19}$, alterações em índices que garantem a topologia de mundo pequeno ${ }^{20}$, redução dos hubs ${ }^{19,21}$, além de decréscimo da conectividade funcional ${ }^{22}$.

A DA representa a principal forma de demência na população idosa, sendo responsável por 60 a $80 \%$ dos casos $^{23}$. Instala-se de modo lento e progressivo, com alterações neuropatológicas, deposição amiloide e emaranhados neurofibrilares de tau hiperfosforilada, que se alojam décadas antes da apresentação clínica da doença ${ }^{24,25}$. Até o momento, o diagnóstico definitivo é dado apenas pós-morte, através da análise anatomopatológica e histológica do cérebro do paciente, sendo necessárias pesquisas para o desenvolvimento de marcadores que possibilitem diagnóstico precoce ${ }^{23}$.

Publicações anteriores, que avaliaram assimetria cerebral em indivíduos com DA e no comprometimento cognitivo leve, relataram alterações na assimetria do volume cortical ${ }^{1}$, diferenças na conectividade funcional ${ }^{26} \mathrm{e}$ em propriedades da substância branca ${ }^{8}$. Além disso, em indivíduos portadores de homozigose para o gene APOE-e4, que representa um fator de risco para o desenvolvimento do Alzheimer, tem sido demonstrada maior propensão à apresentação de um perfil cognitivo assimétrico. ${ }^{27}$

O presente estudo visa a avaliar, de modo exploratório, a assimetria hemisférica funcional, considerando padrões de homofilia (conexões no mesmo hemisfério) e heterofilia (conexões entre os hemisférios) em redes funcionais dinâmicas de portadores de DA, além de analisar a assimetria da conectividade funcional, tendo em vista o processo de progressão da enfermidade.

\section{METODOLOGIA}

Os dados utilizados no presente trabalho são de caráter secundário, tendo sido previamente empregados no estudo de Yang et al. ${ }^{28}$, com a aprovação do Conselho de Revisão Institucional do Hospital Geral de Veteranos de Taipei, Taiwan, para a realização de análises clínicas retrospectivas dos dados clínicos e eletroencefalografia (EEG) dos pacientes. Participaram da análise 123 indivíduos, dentre os quais 108 apresentavam algum grau de Alzheimer, 59 mulheres e 49 homens, com idade média de $78(8,6)$ anos, sendo os estágios da doença: DA muito leve $(\mathrm{N}=15)$, DA leve $(\mathrm{N}=69), \mathrm{DA}$ moderado a grave $(\mathrm{N}=24)$. $\mathrm{O}$ grupo de controle foi composto por 15 indivíduos saudáveis, com idade média de $69,9(9,5)$ anos. Neste trabalho, o grupo de Alzheimer leve apresenta 68 indivíduos, e o de DA moderado a grave, 23 indivíduos, conforme os dados cedidos.

A coleta foi realizada na Clínica de Demência do Instituto Neurológico do Hospital Geral de Veteranos em Taipei, Taiwan. O protocolo de coleta de EEG começou com 5 min de habituação para o exame do ambiente, seguido de três sessões com os olhos fechados, de 10 a 20 segundos, e uma sessão de fotoestimulação. Foram utilizados 19 eletrodos (Fp1, Fp2, F7, F3, Fz, F4, F8, T3, 
$\mathrm{C} 3, \mathrm{Cz}, \mathrm{C} 4, \mathrm{~T} 4, \mathrm{~T} 5, \mathrm{P} 3, \mathrm{Pz}, \mathrm{P} 4, \mathrm{~T} 6, \mathrm{O} 1, \mathrm{e}$ O2) posicionados de acordo com o Sistema Internacional de Posicionamento de Eletrodos (SIPE), o sistema 10/20, com referência auricular, taxa amostral de $256 \mathrm{~Hz}$, filtro passa alta de $0,5 \mathrm{~Hz}$ e filtro passa baixa $70 \mathrm{~Hz}$, "notch filter" de $60 \mathrm{~Hz}$, impedância mantida a $3 \Omega$ Yang et al. ${ }^{28}$

A partir do banco de dados, redes variantes no tempo foram geradas com o objetivo de comparar os padrões de assimetria hemisférica funcional, além da assimetria da conectividade funcional, do ponto de vista dinâmico, considerando os diferentes estágios da doença.

Durante o processo de construção das redes funcionais dinâmicas, os eletrodos foram considerados os nós da rede, e a conexão entre eles foi computada a partir do método dos motifs. Foram geradas redes variantes no tempo e redes estáticas agregadas. Por fim, índices da rede, grau ponderado do nó e E-I index foram analisados à luz da teoria dos grafos.

A análise dos motifs, elaborada por Rosário et al. ${ }^{29}$, permite a avaliação dos níveis de sincronia entre as séries temporais de eletrodos, a partir da contagem de micropadrões existentes no traçado dentro de uma janela de tempo móvel. Para cada instante de tempo com o deslocamento da janela é gerada uma matriz de adjacência que demonstra a estrutura de conectividade do grafo. O deslizamento da janela permite a geração de várias matrizes de adjacência e, consequentemente, de diversas redes. Tais redes são denominados grafos variantes no tempo e permitem a análise cerebral sob a perspectiva dinâmica, enquanto o somatório das matrizes de adjacência de cada grafo forma a Rede Estática Agregada (REA), que possibilita a avaliação do comportamento do cérebro sob o aspecto estático.

\section{RESULTADOS}

Neste trabalho, foi realizada a avaliação de padrões de assimetria hemisférica funcional através da verificação de homofilia e heterofilia de conexões funcionais para diferentes estágios de DA e para o grupo de controle. Além disso, foi avaliado o grau de lateralização da conectividade funcional, considerando a progressão da enfermidade.

A medida escolhida para avaliar a assimetria cerebral das redes funcionais foi o E-I index, análise originalmente proposta por Krackhardt e Stern ${ }^{30}$ para avaliar a proporção de interações entre indivíduos do mesmo grupo e de grupos distintos. Esse índice pode variar de -1 a +1 , com valores negativos que representam homofilia e valores positivos que são indicadores de heterofilia. O E-I index é obtido a partir da razão entre a diferença do número de ligações externas (EL) e internas (IL) com o total de ligações na rede:

$$
\text { E-I index }=(E L-I L) ~ /(E L+I L) \text {. }
$$

A avaliação da lateralização da conectividade funcional, por sua vez, foi realizada a partir da média do grau ponderado dos nós em cada um dos hemisférios.

Tendo em vista que, durante a avaliação de assimetria hemisférica e a análise da assimetria para a conectividade funcional, os dados não apresentaram variâncias iguais, como foi demonstrado pelo teste de Levene, o teste de significância adotado para a avaliação da existência de diferenças significativas entre os grupos foi o de Kruskal-Wallis, com um $p$-valor $<0,05$. As análises estatísticas foram realizadas a partir do pacote estatístico Origin 2018.

A avaliação de assimetria funcional, a partir de padrões de homofilia e heterofilia, demonstrou que os indivíduos de todos os grupos apresentaram um padrão de homofilia mais elevado do que de heterofilia (Figura 1), revelando maior conectividade entre ligações dentro do mesmo hemisfério do que entre hemisférios. Os níveis de homofilia tenderam a diminuir com o avanço da doença, considerando-se os estágios iniciais (DA muito leve e DA leve) com um aumento para o último estágio, sem ultrapassar o grupo de controle (o que pode ser visualizado na Tabela 1). Contudo a análise estatística realizada através do teste Kruskal-Wallis revelou que tais diferenças entre os grupos não são significativas ( $K-W$ qui-quadrado $=3,145$; graus de liberdade $=3 ; \mathrm{P}$-valor $=0,369$ ) .

Figura 1 - Gráfico representando o padrão de homofilia mais elevado do que o de heterofilia para todos os grupos.

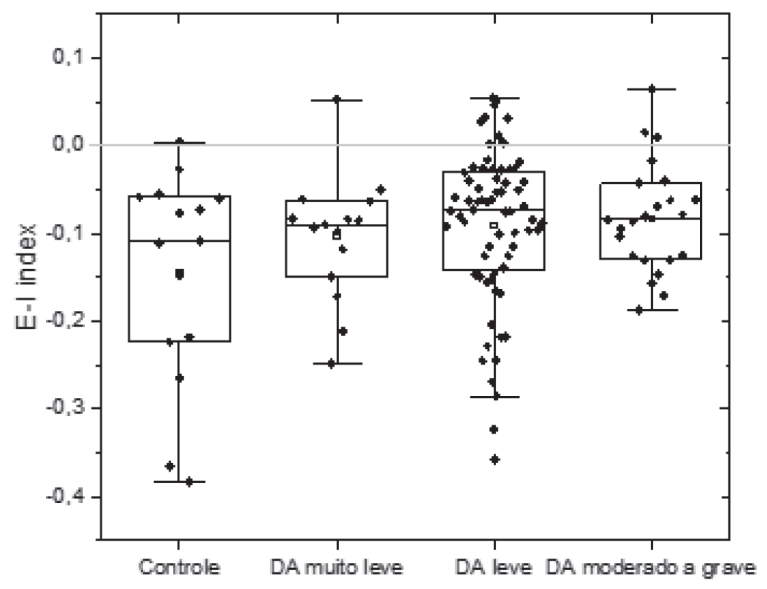

Fonte: Autoria própria 
Tabela 1 - Assimetria hemisférica por padrões de homofilia e heterofilia

\begin{tabular}{|c|c|c|c|c|c|c|c|c|}
\hline \multirow{2}{*}{ Variável } & \multirow{2}{*}{$\mathbf{N}$} & \multicolumn{2}{|c|}{ Min. } & \multicolumn{2}{|c|}{ Max. } & \multirow{2}{*}{ P25 } & \multirow{2}{*}{ Mediana } & \multirow{2}{*}{ P75 } \\
\hline & & Homofilia & Heterofilia & Homofilia & Heterofilia & & & \\
\hline Controle* & 15 & -0.383 & & -0.027 & & $-0,223$ & $-0,108$ & $-0,058$ \\
\hline DA ml & 15 & -0.248 & 0.052 & -0.050 & 0.071 & $-0,149$ & $-0,090$ & $-0,063$ \\
\hline DA leve & 68 & -0.358 & 0.002 & -0.017 & 0.053 & $-0,144$ & $-0,072$ & $-0,028$ \\
\hline DA mg & 23 & -0.187 & 0.009 & -0.017 & 0.063 & $-0,129$ & $-0,083$ & $-0,043$ \\
\hline
\end{tabular}

*Controle - O grupo de controle apresentou apenas um valor para heterofilia, por isso não há como definir mínimo e máximo. Tal valor foi 0.003. P25 - Percentil 25; P75 - Percentil 75; DA ml - DA muito leve; DA mg - DA moderado a grave.

Fonte: Dados da pesquisa

A avaliação da assimetria da conectividade funcional demonstrou uma tendência à redução do índice avaliado para todos os estágios de DA, quando comparada à do grupo de controle (Tabela 2 ). No que diz respeito à conectividade no nível de hemisfério entre os estágios da doença, fases iniciais do Alzheimer e o grupo de controle mostraram propensão a maior conectividade no hemisfério direito, o que foi contrário para o último estágio da doença. A fim de demonstrar a variação entre os hemisférios, foi realizada a diferença da conectividade média para os hemisférios (HD-HE), como é apresentado na Figura 2. Esse resultado demonstra maior disparidade para o estágio moderado a grave. A avaliação estatística realizada a partir do teste Kruskal-Wallis revelou a inexistência de diferenças significativas entre os grupos ( $\mathrm{K}-\mathrm{W}$ qui-quadrado $=1,067$; graus de liberdade $=7$; P-valor $=0,993$ ).
Figura 2 - Diferença hemisférica média da conectividade funcional (em módulo), onde DA ml - DA muito leve; DA mg - DA moderado a grave.

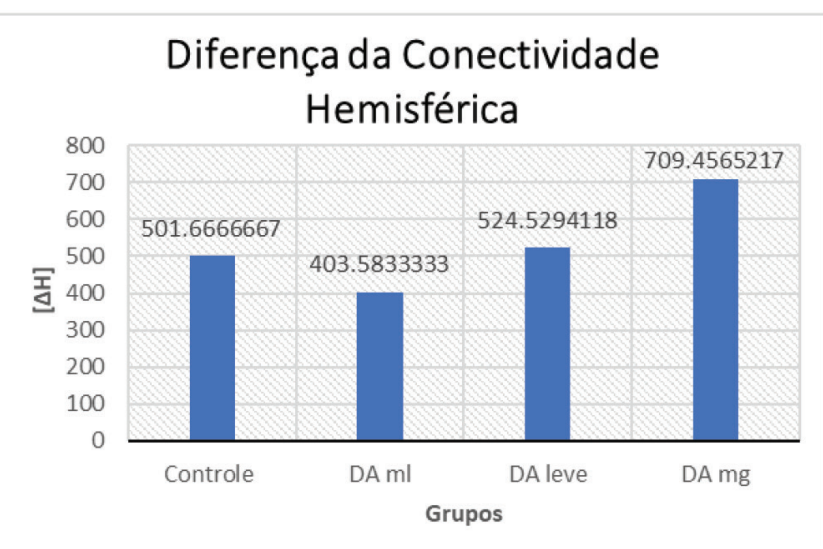

Fonte: Autoria própria.

Tabela 2 - Assimetria hemisférica para a conectividade funcional

\begin{tabular}{|c|c|c|c|c|c|c|c|c|c|c|c|}
\hline \multirow[t]{2}{*}{ Variável } & \multirow{2}{*}{$\mathbf{N}$} & \multicolumn{2}{|c|}{ Min } & \multicolumn{2}{|c|}{ Max } & \multicolumn{2}{|c|}{ P25 } & \multicolumn{2}{|c|}{ Mediana } & \multicolumn{2}{|c|}{ P75 } \\
\hline & & H. D. & H. E. & H. D. & H. E. & H.D. & H.E. & H. D. & H. E. & H.D. & H. E. \\
\hline Controle & 15 & 1702.50 & 1702.87 & 8152.37 & 7650.9 & 2524.12 & 2675.62 & 3541.62 & 3200.12 & 4201.62 & 4142.50 \\
\hline DA ml & 15 & 1122.37 & 1386.50 & 6938.6 & 7402.0 & 2104.0 & 2262.25 & 3356.37 & 3222.50 & 5501.87 & 4664.62 \\
\hline DA leve & 68 & 935.75 & 933.12 & 18676.50 & 21732.3 & 2191.06 & 1948.71 & 2907.93 & 2743.50 & 5157.12 & 5298.75 \\
\hline DA mg & 23 & 1117.88 & 1314.25 & 9068.0 & 7041.62 & 2028.0 & 1938.50 & 2570.87 & 3467.87 & 4303.75 & 4400.125 \\
\hline
\end{tabular}

P25 - Percentil 25; P75 - Percentil 75; H. E - Hemisfério Esquerdo; H.D - Hemisfério Direito; DA mI - DA muito leve; DA mg - DA moderado a grave.

Fonte: Dados da pesquisa.

Os resultados das análises realizadas neste trabalho apontam a propensão a alterações no perfil da assimetria hemisférica funcional em níveis de homofilia das redes e da assimetria da conectividade funcional para os pacientes com Alzheimer.

\section{DISCUSSÃO}

Neste trabalho, foi feita uma comparação da as- simetria hemisférica funcional e da assimetria para a conectividade funcional de redes funcionais corticais dinâmicas em pacientes com Alzheimer, considerando-se os diferentes estágios da doença, em comparação com um grupo de controle. O estudo propiciou a avaliação do índice de conectividade média por nó para os hemisférios cerebrais e a análise dos padrões de homofilia e heterofilia hemisférica, verificando a preferência na formação de conexões. Embora não tenham sido 
encontradas diferenças significativas entre os grupos, os resultados mostraram tendências que assinalam alterações no padrão de homofilia e na assimetria da conectividade funcional.

A teoria da homofilia, estabelecida dentro das ciências sociais, considera que interações ocorrem com maior frequência entre indivíduos semelhantes do que entre os diferentes ${ }^{31}$. Modelos gerativos do conectoma humano têm demonstrado que aspectos próprios da organização de redes funcionais cerebrais podem ser formados a partir do desenvolvimento de ligações homofílicas ${ }^{32,33}$. A avaliação da assimetria cerebral funcional, sob a perspectiva da análise de homofilia, pode ser relevante para a compreensão do mecanismo de segregação da rede, característica que garante uma eficiência local no processamento da informação. Tal eficiência reflete a habilidade da rede em tolerar falhas ${ }^{8,10}$.

Nosso trabalho demonstrou uma tendência à redução geral da homofilia para os diferentes estágios de DA quando comparada à do grupo de controle. Tendo em vista que hubs são preferencialmente afetados na $\mathrm{DA}^{19-21}$, e que há uma tendência à redução da homofilia para as redes funcionais dos pacientes, é provável que essa diminuição seja proveniente, em parte, do ataque aos hubs, sendo necessários trabalhos futuros que avaliem o padrão de homofilia dos hubs de redes funcionais dinâmicas. Nossos resultados também apontaram um leve aumento da homofilia para o último estágio da doença, o que pode ser explicado, parcialmente, por um mecanismo compensatório decorrente do aumento da atrofia do corpo caloso.

O corpo caloso é a principal comissura inter-hemisférica e apresenta alteração já na fase prodrômica da doença ${ }^{34,35}$. Fabri et al. ${ }^{36}$ demonstraram que propriedades estruturais do corpo caloso apresentam importância central para o desenvolvimento e a extensão das assimetrias hemisféricas funcionais. O'Reilly et al. ${ }^{37}$ apontaram, em um estudo de conectividade funcional com modelo animal, antes e depois da secção do corpo caloso, que, a depender da região removida, os níveis de conectividade funcional poderiam ser mantidos por compensação através das áreas não lesionadas, com um aumento da conectividade intra-hemisférica pós-lesão.

A avaliação da assimetria para a conectividade funcional, por sua vez, demonstrou tendência a uma queda do índice entre os diferentes estágios de DA, quando comparada à do grupo de controle. Essa diminuição na conectividade funcional é prevista na literatura, em estudos de redes, a partir de exames de neuroimagem, eletroencefalografia e magnetoencefalografia e pode ser atribuída à neuropatologia da doença ${ }^{20}$. Estudos prévios têm apontado o Alzheimer como uma síndrome de desconexão, com redução da conectividade funcional ${ }^{21}$, distúrbios em circuitos neuronais ${ }^{38} \mathrm{e}$ alterações na sincronia neurona ${ }^{25}$, resultantes de alterações neuropatológicas que levam à perda sináptica, morte neuronal e atrofia cortical $^{20,24}$.

Entre os grupos, para o controle e os estágios iniciais de DA, foi demonstrada uma tendência a maior conectividade no hemisfério direito, diferentemente do que é observado no estágio mais avançado da doença A presença de maior conectividade no hemisfério direito pode ser explicada, em parte, pelo fato de que o hemisfério esquerdo, além de ser acometido primariamente, sofre maiores níveis de atrofia do que o hemisfério direito ${ }^{39,40}$.

\section{LIMITAÇÕES}

Embora padrões de homofilia tenham demonstrado redução, uma avaliação em nível de hubs seria relevante para avaliar se isso ocorre em decorrência da perda deles, uma vez que são preferencialmente afetados durante a evolução da doença. Contudo isso seria computacionalmente inviável no momento.

\section{CONCLUSÃO}

O presente estudo revelou uma tendência à alteração no padrão típico de homofilia cerebral, em âmbito global, com o avanço do Alzheimer para as redes funcionais cerebrais avaliadas sob a perspectiva dinâmica. Além disso, foi demonstrada uma propensão à redução geral da conectividade funcional nos pacientes com a doença, com maiores índices de conectividade para o hemisfério direito. Embora os resultados não tenham apresentado diferenças significativas, provavelmente em virtude da alta variabilidade individual, a presença das tendências sinaliza a necessidade de novos estudos que façam a avaliação da assimetria hemisférica funcional e de assimetria na sincronização hemisférica a partir de análises longitudinais.

\section{AGRADECIMENTOS}

Agradeço aos colegas do Núcleo de Inovação Tecnológica em Reabilitação e do NeuroRedes o apoio durante o trabalho. Em especial, agradeço ao professor José Garcia Vivas Miranda o auxilio durante o processo de elaboração do trabalho.

\section{REFERÊNCIAS}

1. DERFLINGER, S. et al. Grey-matter atrophy in Alzheimer's disease is asymmetric but not lateralized. J. alzheimers dis., Amsterdam, v. 25, n. 2, p. 347, 2011.

2. GOLDBERG, E. et al. Hemispheric asymmetries of cortical volume in the human brain. Cortex, Varese, v. 49, n. 1, p. 200-210, 2013.

3. OCKLENBURG, S. et al. Intrahemispheric white matter asymmetries: the missing link between brain structure and functional lateralization? Rev. Neurosci., London, v. 27, n. 5, p. 465-480, 2016.

4. TOGA, A. W.; THOMPSON, P. M. Mapping brain asymmetry. Neuroscience, London, v. 4, n. 1, p. 37, 2003.

5. LIU, H. et al. Changes in brain lateralization in patients with mild cognitive impairment and Alzheimer's disease: A resting-state functional magnetic resonance study from Alzheimer's disease neuroimaging initiative. Frontiers in neurology, [s.I], v. 9, p. 3, 2018.

6. CABEZA, R. Hemispheric asymmetry reduction in older adults: the Harold model. Psychol. aging, Arlington, v. 17, n. 1, p. 85, 2002. 
7. SUN, Y. et al. Asymmetry of hemispheric network topology reveals dissociable processes between functional and structural brain connectome in community-living elders. Front. aging neurosci., [s.I], v. 9, p. 361, 2017.

8. YANG, C. et al. The abnormality of topological asymmetry between hemispheric brain white matter networks in Alzheimer's disease and mild cognitive impairment. Front. aging neurosci., [s.l], v. 9, p. 261, 2017.

9. OCKLENBURG, S. et al. Lateralization and cognitive systems. Front. Psysiol., Lausanne, v. 5, p. 1143, 2014.

10. REIJNEVELD, J. C. et al. The application of graph theoretical analysis to complex networks in the brain. Clin. Neurophysiol., Limerick, v. 118, n. 11, p. 2317-2331, 2007.

11. MUTLU, A. Y.; BERNAT, E.; AVIYENTE, S. A signal-processing-based approach to time-varying graph analysis for dynamic brain network identification. Comput. Math Method. M., [s.I], v. 2012, 2012.

12. WANG, H. et al. Time-Variant Graph Classification. IEEE Transactions on systems, man, and cybernetics: systems, 2018.

13. SUN, Y. et al. Reduced hemispheric asymmetry of brain anatomical networks is linked to schizophrenia: a connectome study. Cerebral Cortex, New York, v. 27, n. 1, p. 602-615, 2015.

14. SHAH, P. et al. Structural and functional asymmetry of medial temporal subregions in unilateral temporal lobe epilepsy: A 7T MRI study. Human brain mapp., New York, v. 40, n. 8, p. 2390-2398, 2019.

15. LI, D. et al. Reduced hemispheric asymmetry of brain anatomical networks in attention deficit hyperactivity disorder. Brain imaging behave., Springer, p. 1-16, 2018.

16. THOMAS, N. A.; LOETSCHER, T.; NICHOLLS, M. E. R. Asymmetries in attention as revealed by fixations and saccades. Exp. Brain Res., Berlin, v. 232, n. 10, p. 3253-3267, 2014.

17. HABIB, R.; NYBERG, L.; TULVING, E. Hemispheric asymmetries of memory: the HERA model revisited. Trends cogn. sci., Kidligton, v. 7, n. 6, p. 241-245, 2003.

18. SHANG, W. et al. Hemispheric Asymmetry of the Functional Brain Connectome. International Conference on Cognitive Systems and Signal Processing. Springer, Singapore, p. 541-547, $2016 .$.

19. KABBARA, A. et al. Reduced integration and improved segregation of functional brain networks in Alzheimer's disease. J. neural eng., Bristol, v. 15, n. 2 , p. $026023,2018$.

20. BRIER, M. R. et al. Functional connectivity and graph theory in preclinical Alzheimer's disease. Neurobiol. aging, New York, v. 35, n. 4, p. 757-768, 2014.

21. DAI, Z. et al. Identifying and mapping connectivity patterns of brain network hubs in Alzheimer's disease. Cerebral cortex, New York, v. 25, n. 10, p. 3723-3742, 2015.

22. ENGELS, M. M. A et al. Declining functional connectivity and changing hub locations in Alzheimer's disease: an EEG study. BMC neurol., London, v. 15, n. 1, p. 145, 2015.

23. GAUGLER, J. et al. 2019 Alzheimer's disease facts and figures. Alzheimers Dement., Orlando, v. 15, n. 3, p. 321-387, 2019.
24. SERRANO-POZO, A. et al. Neuropathological alterations in Alzheimer disease. Cold Spring Harbor Perspect. Med., Estados Unidos, v. 1, n. 1, p. a006189, 2011.

25. PALOP, J. J.; MUCKE, L. Network abnormalities and interneuron dysfunction in Alzheimer disease. Nat. Rev. Neurosc., London, v. 17, n. 12, p. 777, 2016.

26. WANG, Z. et al. Interhemispheric functional and structural disconnection in alzheimer's disease: a combined resting-state fmri and dti study. PLos ONE, v.10, n.5, p. e0126310, 2015. DOI: 10.1371/journal. pone.0126310.

27. ALVERSON, W. A.; MASSMAN, P. J.; DOODY, R. S. Prevalence and correlates of cognitive asymmetry in a large sample of Alzheimer's disease patients. J. Clin. Exp. Neuropsychol., Lisse, v. 38, n. 5, p. 516-526, 2016.

28. YANG, A. C. et al. Cognitive and neuropsychiatric correlates of EEG dynamic complexity in patients with Alzheimer's disease. Progr. NeuroPsychopharmacol. Biol. Psychiatry, Inglaterra, v. 47, p. 52-61, 2013.

29. ROSÁRIO, R. S. et al. Motif-Synchronization: A new method for analysis of dynamic brain networks with EEG. Physica A, [s.I], v. 439, p. 7-19, 2015.

30. KRACKHARDT, D.; STERN, Robert N. Informal networks and organizational crises: An experimental simulation. Soc. Psychol. Q., Albany, p. 123-140, 1988.

31. MCPHERSON, M.; SMITH-LOVIN, L.; COOK, J. M. Birds of a feather: Homophily in social networks. Annu. Rev. Social., Palo Alto, v. 27, n. 1, p. 415-444, 2001.

32. VÉRTES, P. E. et al. Simple models of human brain functional networks. Proc. Natl Acad. Sci. U. S. A., Washington, v. 109, n. 15, p. 5868-5873, 2012.

33. BETZEL, R. F. et al. Generative models of the human connectome. Neuroimage, Orlando, v. 124, p. 1054-1064, 2016.

34. DI PAOLA, M. et al. When, where, and how the corpus callosum changes in $\mathrm{MCl}$ and $\mathrm{AD}$ : a multimodal MRI study. Neurology, New York, v. 74, n. 14, p. 1136-1142, 2010.

35. WANG, Xu-D. et al. Corpus Callosum atrophy associated with the degree of cognitive decline in patients with Alzheimer's dementia or mild cognitive impairment: a meta-analysis of the region of interest structural imaging studies. J. Psychiat. Res., v. 63, p. 10-19, 2015.

36. FABRI, M. et al. Functional topography of the corpus callosum investigated by DTI and fMRI. World j. radiol, [s.I], v. 6, n. 12, p. 895, 2014.

37. O'REILLY, J. X. et al. Causal effect of disconnection lesions on interhemispheric functional connectivity in rhesus monkeys. Proc. Natl Acad. Sci. U. S. A., Washington, v. 110, n. 34, p. 13982-13987, 2013.

38. BUSCHE, M. A. et al. Tau impairs neural circuits, dominating amyloid- $\beta$ effects, in Alzheimer models in vivo. Threshold, [s.l], v. 30, n. 40, p. $50,2019$.

39. THOMPSON, P. M. et al. Dynamics of gray matter loss in Alzheimer's disease. J. neurosci., Baltimore, v. 23, n. 3, p. 994-1005, 2003.

40. THOMPSON, P. M. et al. Tracking Alzheimer's disease. Ann NY Acad. Sci., New York, v. 1097, p. 183, 2007.

Submetido em: 04/11/2019

Aceito em: 30/11/2019 